05,08, 13

\title{
Особенности поведения зародышей перемагничивания в магнитном поле в одноосных пленках
}

\author{
(C) Р.М. Вахитов ${ }^{1}$, Р.В. Солонецкий ${ }^{1}$, И.Б. Ларионов ${ }^{2}$ \\ ${ }^{1}$ Башкирский государственный университет, \\ Уфра, Россия \\ ${ }^{2}$ Омский государственный университет им. Ф.М. Достоевского, \\ Омск, Россия \\ E-mail: VakhitovRM@rambler.ru
}

(Поступила в Редакцию 23 августа 2016 г.

В окончательной редакции 16 ноября 2016 г.)

\begin{abstract}
Теоретически исследуется влияние магнитного поля на структуру и свойства магнитных неоднородностей, образующихся на дефектах типа „потенциальная яма“ в магнитоодноосных пленках. Показано, что поведение высокоамплитудной и малоамплитудной неоднородностей в магнитном поле существенно различается, и выявлены причины таких различий. Установлено, что в некоторых случаях, когда данные неоднородности в отсутствие поля не существуют, они могут индуцироваться в ненулевом поле. Рассмотрены возможности идентификации обоих типов неоднородностей при их экспериментальном обнаружении.
\end{abstract}

Работа выполнена при поддержке гранта РФФИ (проект № 15-32-51255).

DOI: 10.21883/FTT.2017.06.44481.334

\section{1. Введение}

Известно, что дефекты, присутствующие в магнитных материалах, существенно сказываются на их свойствах [1-3]. В частности, их наличие влияет на фазовые переходы типа спиновой переориентации [4,5], на процессы перемагничивания $[1-3,6,7]$, на динамику доменных границ (ДГ) [8-10], а также на характеристики материалов: на ширину и подвижность ДГ $[8,9]$, на коэрцитивную силу $[3,7]$ и т.п. Кроме того, при определенных условиях дефекты могут генерировать магнитные неоднородности различной топологии: цилиндрические магнитные домены [11], треугольные домены [12], спиральные домены [13], вертикальные блоховские линии [14], а при взаимодействии с движущимися ДГ могут трансформировать их структуру $[15,16]$. При этом структурные неоднородности материала, проявляющие себя как дефекты, могут иметь как естественное происхождение, связанные с несовершенной технологией получения образцов [1], так и быть индуцированными в результате внешнего воздействия, например, импульсным оптическим излучением [14], неоднородными магнитным [12,17] или электрическим [14,18] полями и т.д.

В то же время дефекты различаются не только природой возникновения, но и конфигурацией, размерами и другими характеристиками, а по типу взаимодействия с магнитной системой они могут представлять ,Потенциальный барьер“ или „потенциальную яму“ $[3,19]$. В последнем случае на дефектах при некоторых условиях $[19,20]$ возможно возникновение связанных состояний с магнитной системой, что приводит к образованию на них микромагнитных структур, подстраивающихся под профиль дефектов и соответствующих 0-градусной
ДГ $\left(0^{\circ} Д Г\right)$. Эти неоднородности играют важную роль во многих явлениях, в частности, они могут стать зародышами новой фазы при спин-переориентационных фазовых переходах $[4,5]$, либо доменами обратной намагниченности в процессах перемагничивания материалов $[1-3,21]$. В последнем случае именно с ними связан один из основных механизмов возникновения магнитного гистерезиса, который проявляется в виде задержки и роста зародышей перемагничивания [3]. Однако данный механизм, как и все, что связано со свойствами подобных дефектов, еще мало изучен, несмотря на многочисленные исследования в этой области [1-3,6-8]. Здесь существует множество вопросов, связанных с топологией магнитных неоднородностей, возникающих на такого рода дефектах [13-16], с их особенностями и условиями их образования, дальнейшей их эволюцией, влиянием различных факторов и т.д. В этом плане следует отметить работу [20], в которой были рассмотрены некоторые из них и, в частности, было показано, что $0^{\circ}$ ДГ, зарождающиеся на таких дефектах, могут быть двух типов: малоамплитудные $\left(0^{\circ} Д \Gamma(\mathrm{I})\right)$ и высокоамплитудные $\left(0^{\circ} Д \Gamma(\mathrm{II})\right)$. Они различаются по условиям зарождения и по структуре, причем характеристики $0^{\circ}$ ДГ(I), к которым можно отнести его энергию $E$, ширину $\Delta$ и амплитуду $\theta_{m}$ (максимальный угол отклонения вектора намагниченности М от однородного состояния), по значениям существенно меньше таковых $0^{\circ}$ ДГ(II). Последнее означает, что $0^{\circ} Д Г($ II) энергетически менее выгодны, чем $0^{\circ} Д Г(\mathrm{I})$. Однако согласно расчетам $0^{\circ}$ ДГ(II), образующиеся на достаточно крупных дефектах (когда $L>10 \Delta_{0}$, где $L-$ размер дефекта, $\Delta_{0}-$ характерный размер ДГ $[6,20])$, становятся энергетически более выгодными, чем однородное состояние магнетика. 
В этом случае они будут представлять метастабильные образования, которые могут внести весомый вклад в магнитный гистерезис исследуемых материалов [2,3]. С другой стороны, если учесть размагничивающие поля, обусловленные конечностью образца, то при определенных значениях материальных параметров $0^{\circ}$ ДГ(II) могут стать устойчивыми образованиями [22]. Кроме того, есть определенные экспериментальные данные [23,24], указывающие на реальность их существования. Имеются также достаточные основания считать, что $0^{\circ}$ ДГ(II), зарождающие на двумерных дефектах [24], могут образовывать и спиральные структуры [13]. В свете сказанного становится актуальным продолжение исследований особенностей микромагнитной структуры, возникающей на дефектах типа „потенциальная яма“ одноосного ферромагнетика, и, в частности, выявление ее вклада в процессы перемагничивания образца, что, в свою очередь, представляет и практический интерес [1-3,21].

\section{2. Основные уравнения}

Рассмотрим одноосный ферромагнетик в виде пластины конечной толщины $(D)$, содержащей дефект. Система координат выбирается так, что $O z$ совпадает с осью симметрии кристалла, перпендикулярной поверхности пластины, а ось $O y-$ с направлением, вдоль которого магнетик неоднороден. Соответственно будем считать, что материальные параметры $(A-$ обменный параметр, $K_{u}$ - константа одноосной анизотропии, $M_{s}-$ намагниченность насыщения) зависят только от переменной $y$. Предположим также, что на образец действует внешнее однородное магнитное поле $\mathbf{H} \| O z$. Нетрудно показать, что магнитные неоднородности, возникающие в данном магнетике, будут иметь блоховскую структуру [25]. В этом случае распределение намагниченности М в образце будет определяться лишь одной функцией $\theta=\theta(y)$, где $\theta-$ угол между $\mathbf{M}$ и осью $O z$. Тогда энергию магнетика, приведенную к площади $S$ сечения пластины плоскостью $x \mathrm{Oz}$, можно записать в виде

$E=\int_{-\infty}^{\infty}\left[A(y)\left(\theta^{\prime}\right)^{2}+K_{u}(y) \sin ^{2} \theta-H M_{s}(y) \cos \theta-\varepsilon_{0}\right] d y$

где подынтегральное выражение есть плотность энергии за вычетом энергии однородного состояния $\varepsilon_{0}$ и учитывает вклады обменного взаимодействия, одноосной анизотропии и зеемановского взаимодействия в пренебрежении размагничивающими полями пластины $\left(D \gg \Delta_{0}\right)$. Здесь $\theta^{\prime}-$ производная от угла $\theta$ по переменной $y$.

Уравнение Эйлера-Лагранжа, соответствующее минимуму выражения (1), примет вид

$$
2 \frac{d}{d y}\left(A(y) \theta^{\prime}\right)-2 K_{u}(y) \sin \theta \cos \theta-H M_{s}(y) \sin \theta=0 .
$$

В дальнейшем для определенности рассмотрим дефект типа пластинчатого магнитного включения $[19,20,22]$, в области которого параметры материала меняются скачком

$$
\begin{gathered}
A(y)=\left\{\begin{array}{l}
A_{1},|y|>L / 2 \\
A_{2},|y| \leq L / 2
\end{array}, \quad K_{u}=\left\{\begin{array}{l}
K_{u 1},|y|>L / 2 \\
K_{u 2},|y| \leq L / 2
\end{array},\right.\right. \\
M_{s}=\left\{\begin{array}{l}
M_{s 1},|y|>L / 2 \\
M_{s 2},|y| \leq L / 2
\end{array}\right.
\end{gathered}
$$

где $L-$ размер дефекта. Следует отметить, что, несмотря на простоту выбранной модели дефекта, основные закономерности зарождения магнитных неоднородностей на нем здесь также имеют место [19].

Для того чтобы дефект в одноосном ферромагнетике $\left(K_{u 1}>0\right)$ представлял собой „потенциальную яму“, необходимо выполнение условия: $K_{u 2}<0$. Тогда структура магнитных образований на таких дефектах будет отвечать $0^{\circ}$ ДГ. Соответствующие ей решения уравнения (2), согласно (3), будем искать в виде [20]

$$
\theta=\left\{\begin{array}{l}
\theta_{1}(y),|y|>L / 2 \\
\theta_{2}(y),|y| \leq L / 2
\end{array}\right.
$$

где $\theta_{i}$ - четные функции от $y$ (в силу симметрии задачи), удовлетворяющие уравнениям

$$
A_{i} \frac{d^{2} \theta_{i}}{d y^{2}}-K_{u i} \sin \theta_{i} \cos \theta_{i}-\frac{1}{2} H M_{s i} \sin \theta_{i}=0, \quad i=1,2 .
$$

Исходя из свойств функций $\theta_{i}=\theta_{i}(y)$, решения уравнений (5) достаточно искать в области $y \geq 0$. В этом случае граничные условия для $\theta_{i}(y)$ можно записать в виде

$$
\theta_{1}(y), \theta_{1}^{\prime}(y) \rightarrow 0 \text { при } y \rightarrow \infty, \theta_{2}^{\prime}(0)=0 .
$$

Кроме того будут выполняться условия их „сшивки“ [20]

$$
\theta_{1}(L / 2)=\theta_{2}(L / 2), A_{1} \theta_{1}^{\prime}(L / 2)=A_{2} \theta_{2}^{\prime}(L / 2) .
$$

\section{3. Трансформация структуры $0^{\circ} Д Г$ в магнитном поле}

Первый интеграл уравнений (5) имеет вид

$$
A_{i}\left(\frac{d \theta_{i}}{d y}\right)^{2}-\left[K_{u i} \sin ^{2} \theta_{1}-H M_{s i} \cos \theta_{i}\right]=C_{i},
$$

где $C_{i}-$ константы интегрирования $(i=1,2)$. Из условий (6) следует

$$
C_{1}=H M_{s 1}, C_{2}=-K_{u 2} \sin ^{2} \theta_{2}(0)+H M_{s 2} \cos \theta_{2}(0) .
$$


После интегрирования (8) с учетом (9) для области $y>L / 2$ получим

$$
\theta_{1}(y)=2 \operatorname{arcctg}\left(\sqrt{h_{1} /\left(1+h_{1}\right)} \operatorname{sh}\left(\sqrt{1+h_{1}}\left(y-y_{0}\right) / \Delta\right)\right),
$$

которое по виду отвечает распределению намагниченности в $360^{\circ}$ ДГ [26]. Здесь $h_{1}=H M_{s 1} / K_{u 1}, \Delta_{1}=$ $=\sqrt{\left(A_{1} / K_{u 1}\right.}-$ характерная ширина ДГ в основном объеме кристалла (по существу она совпадает с $\Delta_{0}$ ), $y_{0}$ - некоторая постоянная. Соответственно, для области $0<y<L / 2$ имеем

$\int_{\theta_{2}(y)}^{\theta_{2}(0)} \frac{d \theta}{\sqrt{\sin ^{2} \theta_{2}(0)-\sin ^{2} \theta+h_{2}\left[\cos \theta_{2}(0)-\cos \theta\right]}}=\frac{\overline{\left|K_{u 2}\right|}}{A_{2}} y$,

где $h_{2}=H M_{s 2} /\left|K_{u 2}\right|$. Соотношение (11) можно свести к выражению

$$
F(\varphi(y), k)-F(\varphi(0), k)=y \sqrt{\frac{\left(1+h_{2} \gamma+C_{2} \gamma\right)\left|K_{u 2}\right|}{\left(1-\gamma^{2}\right) A_{2}}},
$$

где $F(\varphi, k)$ - неполный эллиптический интеграл I рода, аргумент $(\varphi)$ и модуль $(k)$ которого определяются соотношениями

$$
\begin{gathered}
\sin \varphi(y)=\frac{\sqrt{1-\gamma^{2}} \sin \theta(y)}{k(1-\gamma \cos \theta(y))}, \\
\left.\left.k=\frac{\frac{\left(1+C_{2}\right)\left(1+\gamma^{2}\right)-2 h_{2} \gamma}{1-h_{2} \gamma+C_{2} \gamma^{2}},}{h_{2}} . C_{2}\right)^{2}\right) \\
\gamma=\frac{\left(1+C_{2}-\sqrt{\left(1+C_{2}\right)^{2}} .\right.}{} .
\end{gathered}
$$

Кроме того из условий (7) и соотношений (8) и (9) вытекает еще одно равенство вида

$$
\begin{aligned}
& \sqrt{\left.\begin{array}{c}
A_{2}\left|K_{u 2}\right|\left[\sin ^{2} \theta_{2}(0)-\sin ^{2} \theta_{2}(L / 2)\right. \\
+
\end{array} h_{2}\left(\cos \theta_{2}(0)-\cos \theta_{2}(L / 2)\right)\right]}= \\
& \quad=\overline{\mid A_{1} K_{u 1}\left[\sin ^{2} \theta_{2}(L / 2)+h_{1}(1-\cos (L / 2))\right]},
\end{aligned}
$$

которое связывает значения $\theta_{2}(y)$ на границе и в центре дефекта.

Полученные соотношения позволяют полностью решить задачу по нахождению распределения намагниченности в области дефекта. Однако из-за их громоздкости такой анализ осуществить крайне сложно, и потому соответствующая задача решалась в основном численно. При этом отметим, что структура $0^{\circ} Д Г$ обоих типов полностью определяется заданием их энергии $E_{s}$ (величина $E$, приведенная к выражению $K_{u 1} \Delta_{1}$ ), амплитуды $\theta_{m}$ (здесь $\left.\theta_{m}=\theta_{2}(0)\right)$ и ширины $\Delta_{s}$. Последняя находится из выражения [27]

$$
\Delta_{s}=2\left[y_{1}-\theta\left(y_{1}\right) / \theta^{\prime}\left(y_{1}\right)\right],
$$

где $y_{1}$ - точка перегиба функции $\theta=\theta(y)$, наиболее удаленная от центра дефекта.
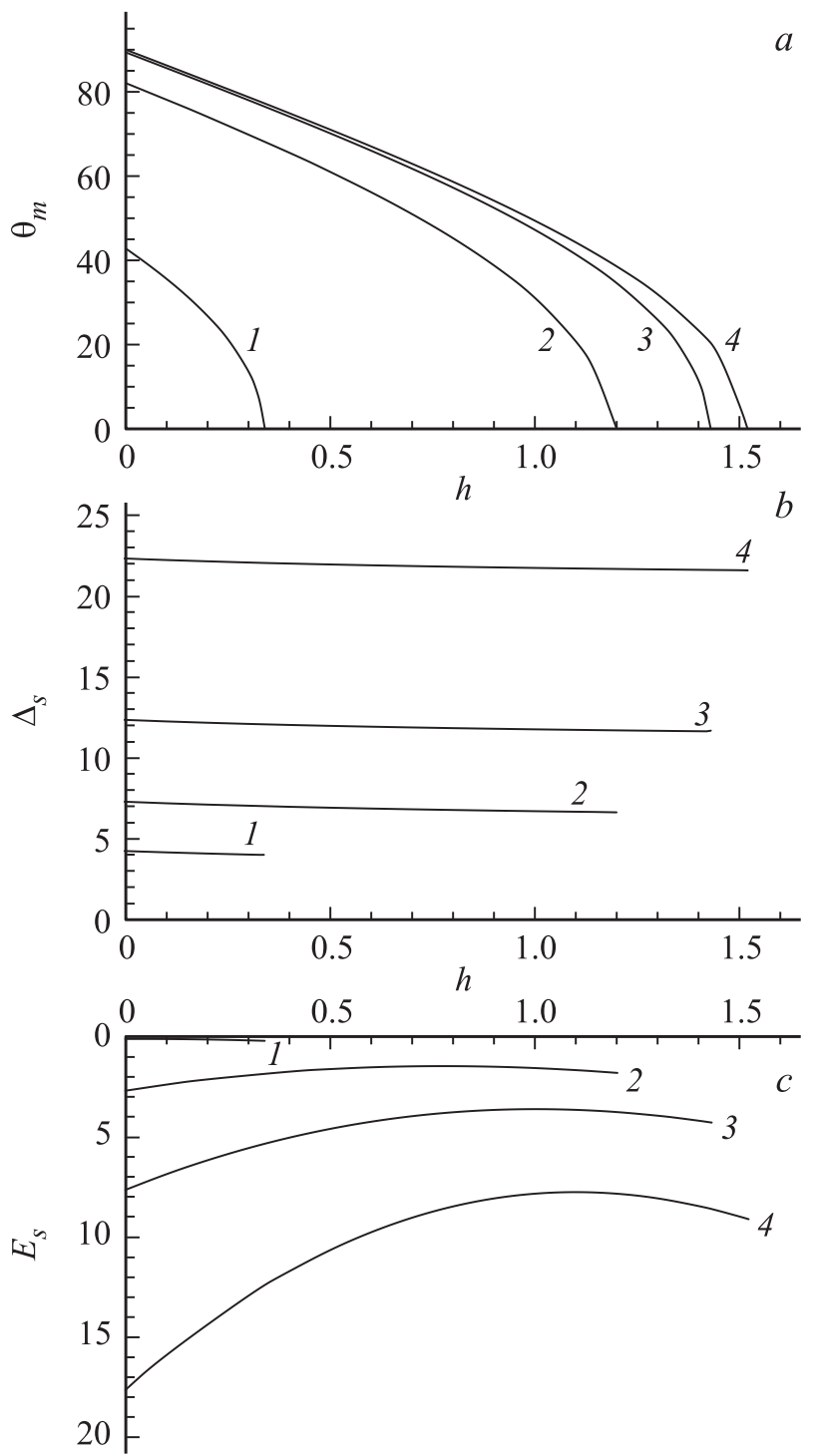

Рис. 1. Зависимости амплитуды $\theta_{m}(a)$, ширины $\Delta_{s}(b)$ и энергии $E_{s}(c) 0^{\circ}$ ДГ(I) от поля при следующих значениях материальных параметров: $K_{u 2}=-K_{u 1}, A_{2}=A_{1}, M_{s 2}=1.3 M_{s 1}$. Линия 1 соответствует $L=2 \Delta_{1}$, линия $2-L=5 \Delta_{1}$, линия $3-L=10 \Delta_{1}$, линия $4-L=20 \Delta_{1}$.

Согласно расчетам магнитные неоднородности, образующиеся на дефектах, в магнитном поле ведут себя неодинаковым образом. С возрастанием величины $h$ (здесь и далее $\left.h=h_{1}\right) 0^{\circ} Д Г(\mathrm{I})$ уменьшается в размерах (рис. 1) вплоть до исчезновения при значениях $h$, превышающих некоторое критическое $h_{c}$. При этом ее ширина $\Delta_{s}$ также уменьшается, но незначительно, в то время как ее амплитуда $\theta_{m}$ уменьшается до нуля $\left(\theta_{m} \rightarrow 0\right)$. $0^{\circ} Д Г(\mathrm{I})$ как бы „испаряется““ (ведет себя подобно капле воды в процессе ее испарения). Процессы перемагничивания $0^{\circ} Д Г(\mathrm{II})$ на дефекте существенно отличаются от рассмотренного сценария. Это связано с тем, что в ее структуре имеется область $\pi / 2<\theta<3 \pi / 2$, в которой магнитные моменты находятся в наиболее невыгодном 


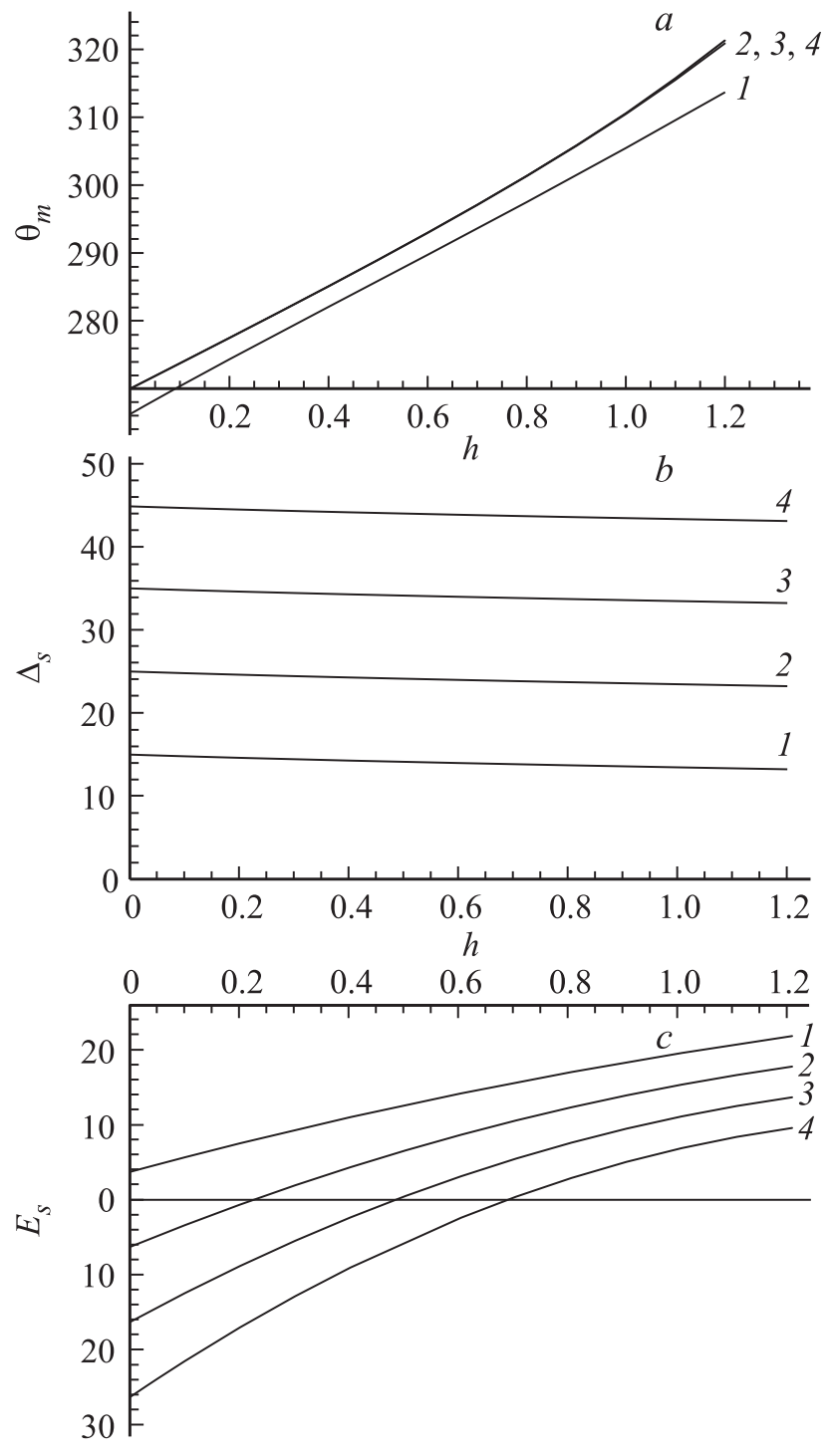

Рис. 2. Зависимости амплитуды $\theta_{m}(a)$, ширины $\Delta_{s}(b)$ и энергии $E_{s}(c) 0^{\circ}$ ДГ(II) от поля при следующих значениях материальных параметров: $K_{u 2}=-K_{u 1}, A_{2}=A_{1}, M_{s 2}=1.3 M_{s 1}$. Линия 1 соответствует $L=10 \Delta_{1}$, линия $2-L=20 \Delta_{1}$, линия $3-L=30 \Delta_{1}$, линия $4-L=40 \Delta_{1}$.

положении по отношению к направлению магнитного поля $\mathbf{H}\left(\varepsilon_{H}=-\mathbf{H M}>0\right)$. Соответственно магнитные моменты в $0^{\circ}$ ДГ(II), принадлежащие данной области, под действием поля Н будут поворачиваться к направлению поля, что приведет к увеличению $\theta_{m}$ (рис. $\left.2, a\right)$ с одновременным уменьшением ширины $0^{\circ}$ ДГ(II) (рис. $\left.2, b\right)$. В пределе, при больших $h$, амплитуда $\theta_{m} \rightarrow 2 \pi$. Отсюда следует (с учетом характера распределения вектора М вне дефекта, определяемого выражением (10)), что $0^{\circ}$ ДГ(II) по структуре будет приближаться к неоднородности, представляющей собой связанное состояние двух $360^{\circ}$ ДГ с противоположными значениями киральности. Последнее, однако, означает, что переход такого связанного состояния в $720^{\circ}$ ДГ (или, по-другому, слияние двух $360^{\circ}$ ДГ) топологически невозможен. В то же время с увеличением $h$ возрастает и энергия $E_{s} 0^{\circ} Д Г(\mathrm{II})$ (рис. 2,c), которая при $h>h_{c}$, где $E_{s}\left(h_{c}\right)=0$, становится положительной. В этом случае однородное состояние магнетика становится энергетически более выгодным, чем его неоднородное состояние. В результате $0^{\circ}$ ДГ(II) становится неустойчивым образованием и коллапсирует.

Однако трансформация $0^{\circ}$ ДГ(II) в поле $\mathbf{H} \| O z$, как показано в [28], может произойти и по другому сценарию, связанному с неустойчивостью структуры $0^{\circ}$ ДГ(II) относительно „неелевских“ флуктуаций вектора М. Это приведет к выходу намагниченности из плоскости стенки, т.е. к ее трансформации в $0^{\circ} Д Г($ II) с квазиблоховской структурой $[25,29]$. Детальный анализ данного механизма перемагничивания $0^{\circ} Д Г($ II) требует отдельного исследования. Тем не менее, можно показать, что при реализации первого механизма процессы перемаг-
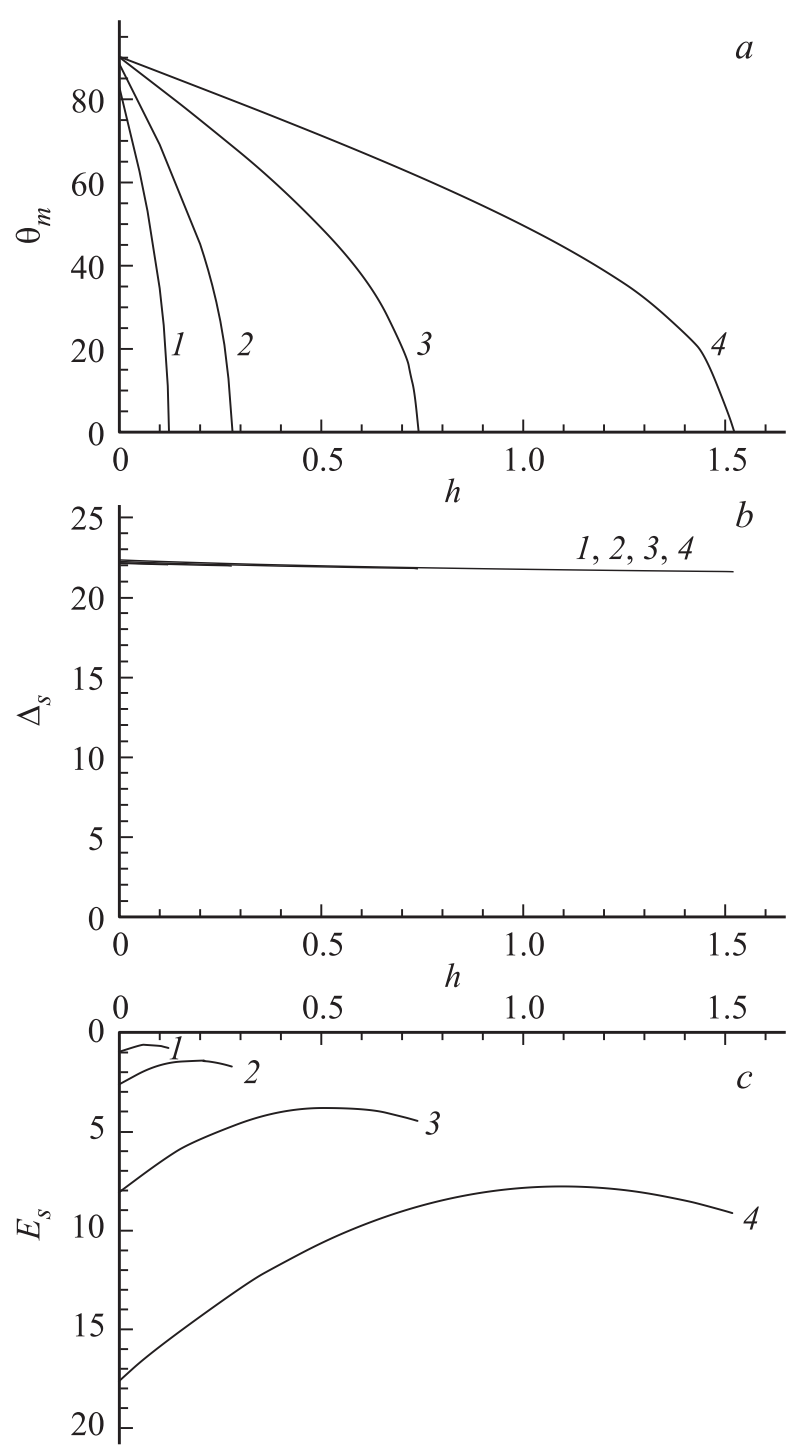

Рис. 3. Зависимости амплитуды $\theta_{m}(a)$, ширины $\Delta_{s}(b)$ и энергии $E_{s}(c) 0^{\circ}$ ДГ(I) от поля при следующих значениях материальных параметров: $L=20 \Delta_{1}, A_{2}=A_{1}, M_{s 2}=1.3 M_{s 1}$. Линия 1 соответствует $K_{u 2}=-0.1 K_{u 1}$, линия $2-K_{u 2}=-0.2 K_{u 1}$, линия $3-K_{u 2}=-0.5 K_{u 1}$, линия $4-K_{u 2}=-K_{u 1}$. 

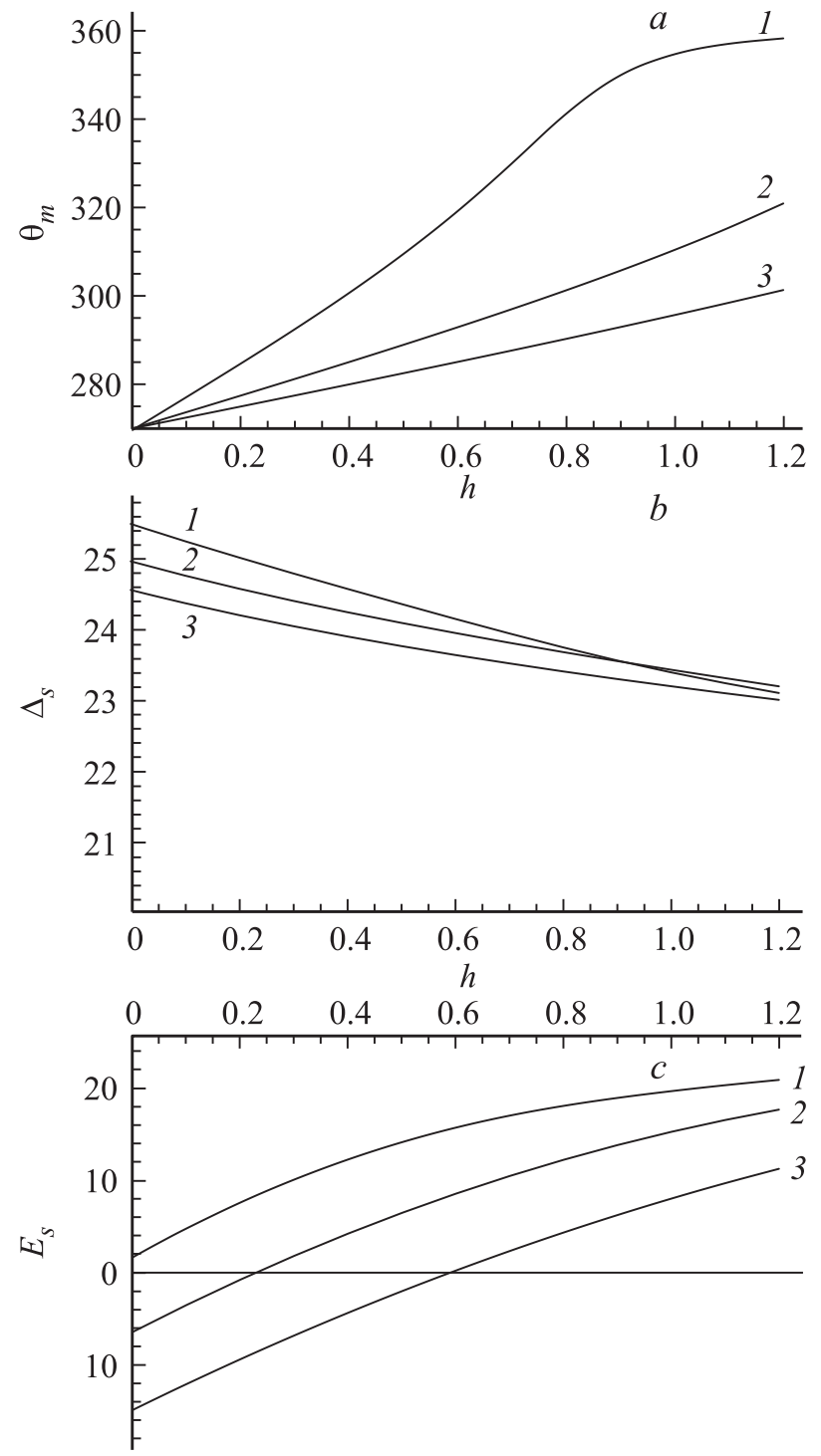

Рис. 4. Зависимости амплитуды $\theta_{m}(a)$, ширины $\Delta_{s}(b)$ и энергии $E_{s}(c) 0^{\circ}$ ДГ(II) от поля при следующих значениях материальных параметров: $L=20 \Delta_{1}, A_{2}=A_{1}, M_{s 2}=1.3 M_{s 1}$. Линия 1 соответствует $K_{u 2}=-0.5 K_{u 1}$, линия $2-K_{u 2}=-K_{u 1}$, линия $3-K_{u 2}=-1.5 K_{u 1}$.

ничивания будут происходить с гистерезисом, а при реализации второго - без гистерезиса. Кроме того, поле $h_{c}$, при котором может иметь место коллапс $0^{\circ} Д Г(\mathrm{II})$ (рис. $2, c)$, значительно меньше поля исчезновения $h_{c}$ $0^{\circ}$ ДГ(I) (рис. 1, $a$ ).

Следует отметить, что характеристики $0^{\circ} Д Г$ обоих типов и критические поля их существования $h_{c}$ сильно зависят от параметров дефекта. В частности, ширина и амплитуда $0^{\circ}$ ДГ обоих типов, а также $h_{c}$, увеличиваются с возрастанием размеров дефекта $L$ (рис. 1,2 ). Причем, если их ширина увеличивается пропорционально величине $L$ (что согласуется с $[20,22]$ ), то амплитуда увеличивается, асимптотически быстро приближаясь к величине $\pi / 2$ для $0^{\circ} Д Г(\mathrm{I})$ или к величине $3 \pi / 2$ для $0^{\circ}$ ДГ(II). Это связано с тем, что область дефекта представляет собой „легкоплоскостной“ магнетик, который создает вращательный момент, заставляющий магнитные моменты повернуться в сторону плоскости $x O y$ : чем значительней размер этой области, тем на больший угол они повернутся. Соответственно требуется большее поле $h$ для преодоления воздействия данного вращательного момента. С другой стороны, используя энергетический подход, можно заметить, что энергия дефекта (которая представляет собой работу, совершаемую магнитным полем по перемагничиванию магнитной неоднородности, закрепленной на дефекте) прямо пропорциональна размерам дефекта: чем больше $L$, тем больше энергия дефекта и, следовательно, тем при больших полях происходит исчезновение $0^{\circ}$ ДГ обоих типов на дефекте.

Аналогичная картина поведения $0^{\circ}$ ДГ обоих типов в магнитном поле имеет место при уменьшении величины $K_{u 2}$, т.е. при увеличении глубины потенциальной ямы. В этом случае с уменьшением $K_{u 2}$ также увеличивается и вращательный момент, заставляющий магнитные моменты „лечь“ в плоскость пластины, что приводит к возрастанию ширины $\Delta_{s}$ и амплитуды $\theta_{m}$ $0^{\circ} Д Г(\mathrm{I})$ (рис. 3). Кроме того, для $0^{\circ} Д Г(\mathrm{I})$ увеличивается $h_{c}$, что также связано с возрастанием энергии дефекта по абсолютной величине: чем глубже потенциальная яма, тем бо́льшая зеемановская энергия нужна для перемагничивания $0^{\circ} Д Г(\mathrm{I})$, закрепленной на дефекте.

Магнитные неоднородности второго типа при умень-

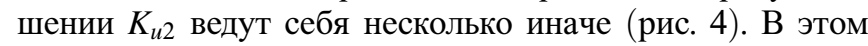
случае увеличивающийся вращательный момент будет поворачивать магнитные моменты к плоскости пластины тем сильнее, чем больше $\left|K_{u 2}\right|$. В итоге амплитуда $\theta_{m}$ для $0^{\circ} Д Г(\mathrm{II})$ будет уменьшаться, так как $\theta_{m}>3 \pi / 2$ и $\theta_{m} \rightarrow 2 \pi$ в результате действия магнитного поля. В то же время уменьшение $\theta_{m}$ с увеличением $\left|K_{u 2}\right|$ приводит в результате к уменьшению ширины $0^{\circ}$ ДГ(II), что согласуется с рис. $4, a$.

\section{4. Устойчивые состояния $0^{\circ}$ ДГ в магнитном поле}

Очевидно, для рассмотрения устойчивых состояний $0^{\circ}$ ДГ в зависимости от материальных параметров и приложенного поля необходимо учесть влияние размагничивающих полей образца, обусловленных его конечностью. Для магнитных неоднородностей блоховского типа вклад размагничивающих полей в энергию магнетика (приведенную к площади $S$ ) (1) определяется выражением вида

$$
\begin{aligned}
E_{m s}= & D^{-1} \int_{-\infty}^{\infty} \int \mid\left[M_{s}(y) M_{s}\left(y^{\prime}\right) \cos \theta(y) \cos \theta\left(y^{\prime}\right)\right. \\
& \left.-M_{s 1}^{2} \cos ^{2} \theta_{0}\right] \ln \left(1+\frac{D^{2}}{\left(y-y_{0}\right)^{2}}\right) \mid d y d y^{\prime},
\end{aligned}
$$

где $\theta_{0}-$ угол, определяющий ориентацию вектоpa $\mathbf{M}$ в доменах $(y \rightarrow \infty)$. Соответствующее уравнение 

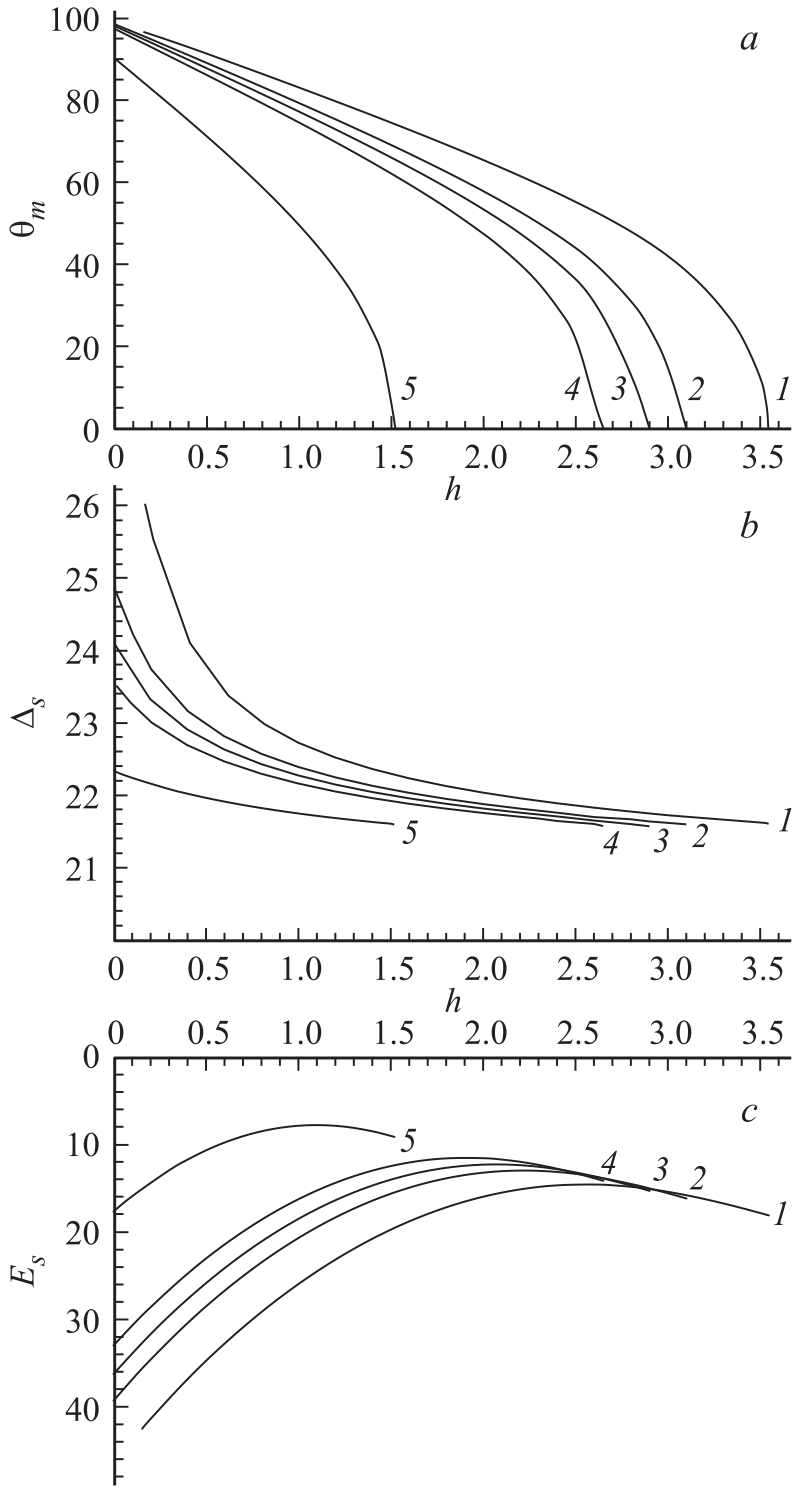

Рис. 5. Зависимости амплитуды $\theta_{m}(a)$, ширины $\Delta_{s}(b)$ и энергии $E_{s}(c) 0^{\circ}$ ДГ(I) от поля при следующих значениях материальных параметров: $L=20 \Delta_{1}, A_{2}=A_{1}, M_{s 2}=1.3 M_{s 1}$, $D=10 \Delta_{1}$. Линия 1 соответствует $Q=1$, линия $2-Q=1.3$, линия $3-Q=1.5$, линия $4-Q=1.8$, линия $5-$ без учета магнитостатики.

Эйлера-Лагранжа будет содержать дополнительное слагаемое, представляющее собой „вариационную“ производную [30] от выражения (16)

$$
\begin{aligned}
\frac{\delta E_{m s}}{\delta \theta} & =-2 D^{-1} M_{s}(y) \sin \theta(y) \\
& \times \int_{-\infty}^{\infty} M_{s}\left(y^{\prime}\right) \cos \theta\left(y^{\prime}\right) \ln \left(1+\frac{D^{2}}{\left(y-y^{\prime}\right)^{2}}\right) d y^{\prime} .
\end{aligned}
$$

В этом случае уравнение, описывающее структуру магнитных неоднородностей в рассматриваемом магнетике, будет являться уже нелинейным интегродифференциальным уравнением второго порядка с переменными коэффициентами. Решение данного уравнения аналитически через известные функции не представляется возможным, поэтому для его решения использовался метод численного интегрирования, в котором применялся метод прогонки с использованием итерационной процедуры по Ньютону [31]. Соответствующая программа вычислений позволила не только найти распределение намагниченности М в области дефекта, но и определить характеристики $0^{\circ}$ ДГ. Из численных расчетов следует, что вклад размагничивающих полей весом и сказывается как на структуре обоих типов
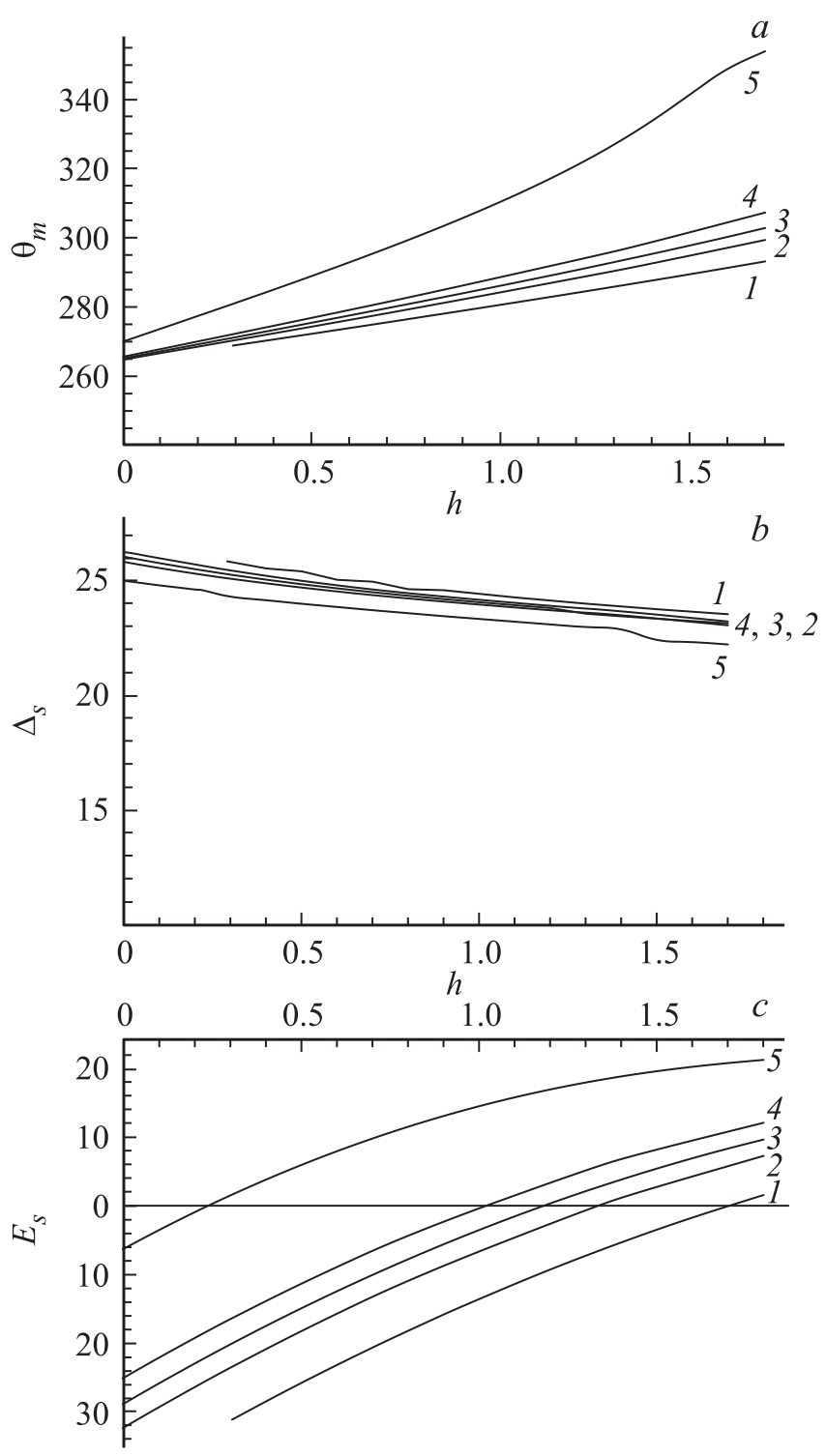

Рис. 6. Зависимости амплитуды $\theta_{m}(a)$, ширины $\Delta_{s}(b)$ и энергии $E_{s}(c) 0^{\circ}$ ДГ(II) от поля при следующих значениях материальных параметров: $L=20 \Delta_{1}, A_{2}=A_{1}, M_{s 2}=1.3 M_{s 1}$, $D=10 \Delta_{1}$. Линия 1 соответствует $Q=1$, линия $2-Q=1.3$, линия $3-Q=1.5$, линия $4-Q=1.8$, линия $5-$ без учета магнитостатики. 


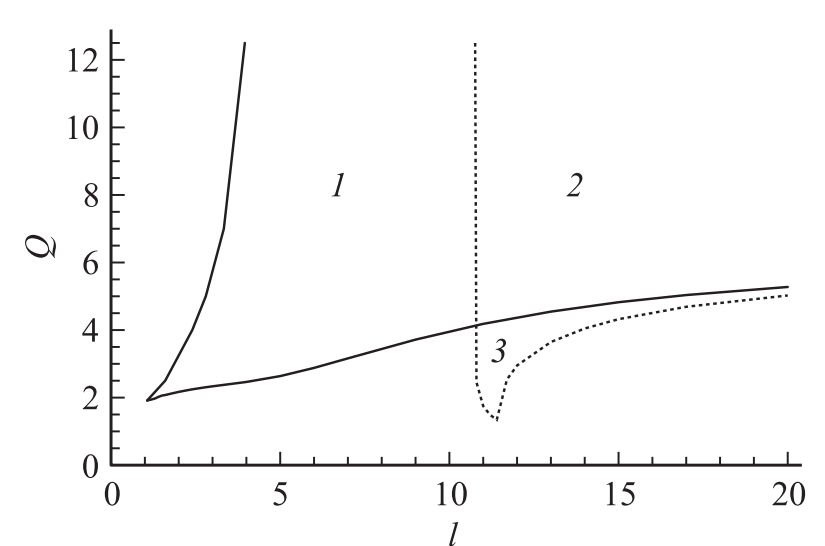

Pис. 7. Диаграмма области существования магнитных неоднородностей типа $0^{\circ}$ ДГ(I) и $0^{\circ}$ ДГ(II) при $K_{u 2}=-0.15 K_{u 1}$, $A_{2}=A_{1}, M_{s 2}=1.3 M_{s 1}, D=10 \Delta_{1}$. Штриховые линии обозначают границу области существования $0^{\circ}$ ДГ(II), а сплошные границу области существования $0^{\circ}$ ДГ(I). Область 1 - это область, в которой существует только $0^{\circ}$ ДГ(I), $2-$ существуют и $0^{\circ}$ ДГ(I) и $0^{\circ}$ ДГ(II), 3 - существует только $0^{\circ}$ ДГ(II).

$0^{\circ}$ ДГ (рис. 5,6), так и на их устойчивых состояниях. В частности, в малых полях амплитуда $\theta_{m} 0^{\circ} Д Г$ обоих типов в незначительной степени зависит от фактора качества $Q\left(Q=K_{u 1} / 2 \pi M_{s 1}^{2}\right)$, а в больших полях существенно. Однако ширина $0^{\circ}$ ДГ в зависимости от ее типа ведет себя по-разному: поведение ширины $0^{\circ} Д Г(\mathrm{I})$ противоположно поведению ее амплитуды, а ширина $0^{\circ}$ ДГ(II) мало зависит от $Q$ во всем интервале полей. В тоже время с уменьшением величины $Q$ размеры $0^{\circ} Д Г(\mathrm{I})$ (ширина $\Delta_{s}$ и амплитуда $\theta_{m}$ ) и критическое поле существования увеличиваются. Аналогичные зависимости от $Q$ имеют место и для характеристик $0^{\circ}$ ДГ(II). Такая зависимость характеристик обоих типов $0^{\circ}$ ДГ от $Q$ объясняется тем, что размагничивающие поля, энергия которых обратно пропорциональна $Q$ [22], стремятся развернуть магнитные моменты в плоскость пластины: чем меньше $Q$, тем сильнее такое воздействие. При $Q=1$ оба типа $0^{\circ} Д Г$ в нулевом поле не существуют, так как равновесное направление М в доменах в этом случае является неустойчивым. Более того, они не существуют и при включении поля $\mathbf{H} \| O z$; $0^{\circ}$ ДГ становятся устойчивыми лишь при достижении полем некоторого порогового значения $h_{l}$. В этом случае область существования $0^{\circ}$ ДГ обоих типов ограничена значениями поля $h$, лежащими в интервале $h_{l}<h<h_{r}$. Здесь $h_{r}$ - верхнее значение критического поля, при котором $0^{\circ} Д Г$ исчезают. При этом $0^{\circ} Д Г(\mathrm{I})$ исчезает посредством непрерывного уменьшения амплитуды $\left(\theta_{m} \rightarrow 0\right)$, она как бы „испаряется“, а $0^{\circ} Д Г($ II) - путем коллапсирования либо путем перестройки своей структуры с образованием $0^{\circ}$ ДГ(II) с квазиблоховским распределением намагниченности [28]. Следует отметить, что нижнее значение фактора качества $Q$, при котором в нулевом поле $0^{\circ} Д Г$ еще существует (при данных значениях материальных параметров), согласно расчетам соответствует $Q=1.143$. Другие пороговые значения $Q$, при которых $0^{\circ}$ ДГ обоих типов существуют, можно определить из диаграммы устойчивых состояний $0^{\circ}$ ДГ в координатах $Q-l$, где $l=L / \Delta_{1}$ (рис. 7).

Очевидно, что критические поля $h_{c}$, при которых исчезают $0^{\circ}$ ДГ обоих типов, можно ассоциировать с коэрцитивной силой $h_{c}$ исследуемого образца $[2,32]$. С учетом того, что поле $h_{c}$, при котором исчезают $0^{\circ} Д Г(\mathrm{I})$, значительно больше соответствующего поля коллапсирования $0^{\circ} Д Г(\mathrm{II})$, основной вклад в коэрцитивную силу будут вносить $0^{\circ} Д Г(\mathrm{I})$. Однако из диаграммы устойчивых состояний $0^{\circ} Д Г$ (рис. 7) видно, что при некоторых значениях материальных параметров доминирующий вклад в коэрцитивную силу может внести и $0^{\circ} Д Г(\mathrm{II})$. В то же время $0^{\circ} Д Г(\mathrm{I})$ по своим свойствам и поведению в магнитном поле полностью совпадает с $0^{\circ}$ ДГ, используемой для модельного представления доменов обратной намагниченности [32].

\section{5. Заключение}

Таким образом, из приведенных расчетов следует, что два вида магнитных образований на дефектах типа „потенциальная яма“ различаются по структуре и по свойствам. Было установлено, что наличие области, в которой $\varepsilon_{H}=-\mathbf{M H}>0$ в плоскости вращения магнитных моментов $0^{\circ} Д Г($ II), приводит к тому, что $0^{\circ} Д Г($ II), во-первых, существенно отличаются от $0^{\circ} Д Г(I)$ по размерам $[20,22,24]$. Во-вторых, при определенных значениях параметров материала $0^{\circ} Д Г(I I)$ становятся устойчивыми образованиями, т.е. энергетически более выгодными, чем $0^{\circ} Д Г(\mathrm{I})$. В-третьих, при магнитооптическом наблюдении поверхности магнитоодноосных пленок в поляризационном микроскопе в случае, когда присутствующие в них дефекты имеют округлую форму сквозного типа, $0^{\circ} Д Г(\mathrm{II})$, образующиеся на них, будут иметь изображение, повторяющее его профиль и имеющее ореол [24]. В-четвертых, при наложении магнитного поля, параллельного оси симметрии, $0^{\circ} Д Г(\mathrm{II})$, закрепленные на дефектах, перемагничиваются в полях, значительно меньших, чем $0^{\circ} Д Г(\mathrm{I})$. И наконец, в некоторых случаях (когда значения параметров материала таковы, что $0^{\circ} Д Г$ на подобного рода дефектах не образуются) данные неоднородности могут быть индуцированы в ненулевых магнитных полях и существовать в определенном диапазоне значений этих полей. В этом случае их зарождением, эволюцией и уничтожением можно управлять с помощью внешних магнитных полей, что аналогично поведению цилиндрических магнитных доменов в одноосных пленках [30]. Последнее позволяет говорить о возможности технических применений вышеуказанных неоднородностей. 


\section{Список литературы}

[1] Д.Д. Мишин. Магнитные материалы. Высш. шк., М. 1991. $386 \mathrm{c}$.

[2] D. Gall. In: Handbook of magnetism and advanced magnetic matirials. V. 2. Micromagnetism / Eds H. Kronmuller, S. Parkin. John Wiley \& Sons. N.Y. (2007). P. 1023.

[3] Г.С. Кандаурова. Сорос. образоват. журнал 1, 100 (1997).

[4] В.К. Власко-Власов, М.В. Инденбом. ЖЭТФ 86, 1084 (1984).

[5] Р.М. Вахитов, А.Р. Юмагузин. ФТТ 43, 65 (2001).

[6] H. Kronmuller. In: Handbook of magnetism and advanced magnetic matirials. V. 2. Micromagnetism / Eds H. Kronmuller, S. Parkin. John Wiley \& Sons. N.Y. (2007). P. 733.

[7] Y. Sun, R. Gao. Solid State Commun. 149, 393 (2009).

[8] E.A. Périgo, I. Titov, R. Weber, D. Honecker, E.P. Gilbert, M.F. De Campos, A. Michels. J. Alloys Comp. 677, 139 (2016).

[9] Е.Б. Магадеев, Л.С. Успенская, Р.М. Вахитов. Изв. РАН. Сер. физ. 77, 1406 (2013).

[10] И.Ю. Джежеря, М.В. Сорокин. ФТТ 41, 1231 (1999).

[11] Д.П. Куликова, А.П. Пятаков, Е.П. Николаева, А.С. Сергеев, Т.Б. Косых, З.А. Пятакова, А.В. Николаев, А.К. Звездин. Письма в ЖЭТФ 104, 196 (2016).

[12] Л.П. Иванов, А.С. Логгинов, Г.А. Непокойчицкий, И.И. Никитин. ЖЭТФ 88, 260 (1985).

[13] В.В. Федотова, А.П. Гесь, Т.А. Горбачевская. ФТТ 37, 2835 (1995).

[14] А.С. Логгинов, Г.А. Мешков, А.В. Николаев, А.П. Пятаков. Письма в ЖЭТФ 86, 124 (2007).

[15] E.G. Ekomasov, R.R. Murtazin, O.B. Bogomazova, A.M. Gumerov. J. Magn. Magn. Mater. 339, 133 (2013).

[16] Б.Н. Филиппов, М.Н. Дубовик. ФТТ 56, 931 (2014).

[17] M.V. Logunov, S.F. Nikitov, M.V. Gerasimov, F.V. Spirin, A.V. Balyasov. Ultrafast Magnetism I. Proc. Int. Conf., Springer Int. Publ. Switzerland (2015). P. 98.

[18] Г.В. Арзамасцева, А.М. Балбашов, Ф.В. Лисовский, Е.Г. Мансветова, А.Г. Темирязев, А.Г. Темирязева. ЖЭТФ 147, 793 (2015).

[19] Е.Б. Магадеев, Р.М. Вахитов. ТМФ 184, 134 (2015).

[20] Р.М. Вахитов, Е.Б. Магадеев. ФММ 115, 906 (2014).

[21] Y. Zhang, X. Li, X. Zhang, Y. Zhao. J. Magn. Magn. Mater. 408, 228 (2016).

[22] Р.М. Вахитов, Е.Б. Магадеев, А.Р. Юмагузин, Р.В. Солонецкий. ФТТ 57, 1462 (2015).

[23] М.В. Четкин, Ю.Н. Курбатова, Т.Б. Шапаева. ФТТ 52, 1795 (2010).

[24] Р.М. Вахитов, Т.Б. Шапаева, Р.В. Солонецкий, А.Р. Юмагузин. Докл. АН Сер. физ. химия 470, 674 (2016).

[25] Е.Б. Магадеев, Р.М. Вахитов. ФТТ 53, 944 (2011).

[26] М.Я. Широбоков. ЖЭТФ 15, 57 (1945).

[27] A. Hubert, K. Schäfer. Magnetic domains. Springer-Verlag. Berlin (2009). $696 \mathrm{p}$.

[28] H.E. Khodenkov, N.N. Kudelkin, V.V. Randoshkin. Phys. Status Solidi A 84, K135 (1984).

[29] В.В. Плавский, М.А. Шамсутдинов, Б.Н. Филиппов. ФММ 88, 22 (1999).

[30] А. Малоземов, Дж. Слонзуски. Доменные стенки в материалах с цилиндрическими магнитными доменами. Мир, M. (1982). 382 c.

[31] Н.С. Бахвалов, Н.П. Жидков, Г.М. Кобельков. Численные методы. Наука, М. (1987). 600 с.

[32] Р.М. Вахитов, А.Р. Юмагузин. ФММ 106, 477 (2008). 